〔特集〕光機能材料の新展開

Paper

\title{
Synthesis of Gold-Silica Core-Shell Nanoparticles with Tunable Shell Thickness
}

\author{
Yusuke MORIGUCHI, Xiangeng MENG, Koji FUJITA, Shunsuke MURAI and Katsuhisa TANAKA
}

Department of Material Chemistry, Graduate School of Engineering, Kyoto University, Katsura Nishikyo-ku, Kyoto 615-8510, Japan

Received September 3, 2012

\begin{abstract}
A synthesis procedure of gold-silica core-shell nanoparticles has been developed in this work. We have investigated the effects of the amounts of poly(vinylpyrrolidone) (PVP), water, and tetraethoxysilane (TEOS) in the starting materials on the shell thickness. The results reveal that the modification of the surface of gold nanoparticles with PVP plays a critical role in the formation of smooth coating with silica. The shell thickness can be well controlled by changing the amounts of water and TEOS, typically ranging from 2 to $25 \mathrm{~nm}$. We have examined optical extinction properties experimentally and numerically; our experimental results are consistent with the calculations.
\end{abstract}

KEY WORDS

Gold nanoparticles, Core-shell nanoparticles, Localized surface plasmon resonance

\section{Introduction}

Metal nanoparticles could potentially lead to extensive applications in various areas such as nanolasers ${ }^{1)}$, solar cells $^{2)}$, sensors ${ }^{3-5)}$, and magneto-optics ${ }^{6)}$ primarily owing to localized surface plasmon resonance (LSPR). LSPR gives enhanced local electric field in the vicinity of the surface of a metal nanoparticle, which can modify many optical processes. In studies on the modification of spontaneous emissions, both fluorescence enhancement and quenching have been observed for fluorophores in the vicinity of metallic nanostructures ${ }^{7,8)}$. The enhancement factor is largely dependent on the distance between the fluorophore and the metal, because the radiative and nonradiative transitions of fluorophores vary with their spatial separation ${ }^{7}$. As a result, it is usual that the maximum enhancement is obtained not on the metallic surface but in the region a few to tens of nanometers away from the surface $^{8)}$. Therefore, to attain the maximum enhancement, a shell-isolation technique has been widely adopted by inserting a dielectric spacer between a fluorophore and the metal nanostructure ${ }^{9-14)}$.

Among various dielectric shells, silica $\left(\mathrm{SiO}_{2}\right)$ is the most practical and studied material. The $\mathrm{SiO}_{2}$ shell can provide a chemically inert environment for the metal core while keeping the high optical transparency ${ }^{15}$. The general $\mathrm{SiO}_{2}$ coating technique is based on the Stöber method in a system containing metal core nanoparticles ${ }^{16)}$. Namely, a silane precursor is added to the solutions of metal nanoparticles in a mixture of ethanol, water, and catalyst (e.g., ammonia) to cause nucleation of $\mathrm{SiO}_{2}$ on the surface of the particles. Subsequently, hydrolysis and polycondensation of the silane precursor result in the formation of $\mathrm{SiO}_{2}$ shell with controlled thickness. Since gold ( $\mathrm{Au}$ ) and silver ( $\mathrm{Ag}$ ) nanoparticles are not suitable for direct $\mathrm{SiO}_{2}$ coating due to their low chemical affinity to $\mathrm{SiO}_{2}$, Mulvaney $e t$ al. reported a three step procedure for coating Au nanoparticles with $\mathrm{SiO}_{2}$ using a silane coupling agent, aminopropyltrimethoxysilane, as the surface primer ${ }^{17)}$. This method is effective but timeconsuming and not easily controlled. Alternatively, using a nonionic and amphiphilic polymer, poly(vinylpyrrolidone) (PVP), instead of a silane coupling agent as the surface primer, Graf et al. developed a simpler and faster method to coat $\mathrm{Au}$ and $\mathrm{Ag}$ nanoparticles with $\mathrm{SiO}_{2}{ }^{18)}$. A thin shell thickness, less than several tens of nanometers, is usually required for many potential applications, because the enhancement of the electric field occurs only in the vicinity of the surface. Although several research groups have prepared $\mathrm{Au}-\mathrm{SiO}_{2}$ core-shell (Au@ $\mathrm{SiO}_{2}$ ) nanoparticles with $\mathrm{SiO}_{2}$ thickness less than several tens of nanometers, the shell tends to be rough and inhomogeneous and the fine tuning is difficult as the thickness becomes smaller ${ }^{17,19-21)}$.

In this work, we improve the method of Graf et al. to give 
a fast, easy, and adoptable method to coat Au nanoparticles with $\mathrm{SiO}_{2}$ thickness below $25 \mathrm{~nm}$. We have systematically examined the effects of the amounts of PVP, water, and tetrathoxysilane (TEOS) on the shell thickness. Furthermore, we have examined optical extinction properties of the $\mathrm{Au} @ \mathrm{SiO}_{2}$ nanoparticles experimentally and numerically. The experimental results agree with the calculations, confirming the uniform growth of the shell.

\section{Experimental}

\subsection{Materials}

For the preparation of Au nanoparticles, $\mathrm{HAuCl}_{4}$ (Kishida), trisodium citrate (Wako) and distilled water were used as the starting materials. For the preparation of $\mathrm{Au} @ \mathrm{SiO}_{2}$ nanoparticles, the as-prepared Au nanoparticles, TEOS (95\%, Nacalaitesque), PVP (average molar masses of 10 $\mathrm{kg} / \mathrm{mol}$, Sigma-Aldrich), ethanol ( $99 \%$, Nacalaitesque), and ammonia aqueous solution ( $28 \mathrm{wt} \%$, Wako) were used as the starting materials.

\subsection{Synthesis}

Au nanoparticles having an average diameter of $13.5 \mathrm{~nm}$ were synthesized according to the standard sodium citrate procedure ${ }^{22)}$. First, $4 \mathrm{ml}$ of aqueous $\mathrm{HAuCl}_{4}$ solution (10 $\mathrm{mg} / \mathrm{ml}$ ) was added to $96 \mathrm{ml}$ water in reflux at $100^{\circ} \mathrm{C}$ under vigorous stirring. After $10 \mathrm{~min}, 10 \mathrm{ml}$ of aqueous sodium citrate solution $(10 \mathrm{mg} / \mathrm{ml})$ was added into the above solution. The reaction lasted $12 \mathrm{~min}$, and then the colloidal solution was cooled down to room temperature for subsequent purpose.

The outline of the $\mathrm{SiO}_{2}$ coating procedure is shown in Fig. 1. In this study, we performed $\mathrm{SiO}_{2}$ coating by altering the amounts of PVP, $\mathrm{H}_{2} \mathrm{O}$, and TEOS. The composition of the starting solutions is summarized in Table 1. The first step was the functionalization of the surface of $\mathrm{Au}$ nanoparticles with PVP by mixing varied amounts of aqueous PVP solution $(25.6 \mathrm{mg} / \mathrm{ml}, 0.5 \mathrm{ml} \leq x \leq 0.15 \mathrm{ml})$ with $7.0 \mathrm{ml}$ of Au colloidal solution under vigorous stirring for $24 \mathrm{~h}$ at room temperature. The mixture was washed once with acetone by centrifugation $(6,000 \mathrm{rpm})$ for $30 \mathrm{~min}$ and then redispersed in $1.816 \mathrm{ml}$ of ethanol for the purpose of $\mathrm{SiO}_{2}$ coating. An aqueous ammonia solution $(0.084 \mathrm{ml})$ and a varied amount of water $(0.00 \mathrm{ml} \leq y \leq 0.15 \mathrm{ml})$ were added into the Au-PVP/ethanol solution under vigorous stirring. Then, a TEOS/ethanol solution $(10 \mathrm{vol} \%, 0.5 \mathrm{ml}$ $\leq z \leq 0.15 \mathrm{ml}$ ) was added under vigorous stirring. When the amount of TEOS/ethanol solution was more than 0.080 $\mathrm{ml}$, the TEOS/ethanol solution was added stepwise by 0.040 $\mathrm{ml}$ at each step with an interval of $1 \mathrm{~h}$. After the TEOS/ ethanol solution was added completely, the solution was stirred further for $12 \mathrm{~h}$ at room temperature to grow $\mathrm{SiO}_{2}$ shell. The $\mathrm{Au} @ \mathrm{SiO}_{2}$ nanoparticles were separated by centrifugation for $30 \mathrm{~min}$ three times (acetone, $6,000 \mathrm{rpm}$ ). The resultant $\mathrm{Au} @ \mathrm{SiO}_{2}$ nanoparticles were redispersed in water for storage.

\subsection{Sample characterizations}

The morphology of the nanoparticles was characterized with transmission electron microscope (JEM 2100F, operation voltage: $200 \mathrm{keV}$ ). The optical extinction spectra were measured by a JASCO V-570 UV-VIS-NIR spectrophotometer.

\subsection{Numerical simulations}

Optical extinction properties were simulated by a discrete dipole approximation (DDA) method in DDSCAT. DDSCAT is a Fortran code for calculating scattering and absorption of light based on $\mathrm{DDA}^{23,24)}$. In the simulation, we supposed that the refractive index of the surrounding medium of the particle was 1.36 . A linearly polarized light was incident on the particle. The diameter of Au core is $13.5 \mathrm{~nm}$, and the shell thickness was set to be $0,4.2,14.5$, and $25.0 \mathrm{~nm}$. The refractive index of $\mathrm{Au}$ was taken from the literature ${ }^{25)}$ and the refractive index of $\mathrm{SiO}_{2}$ was set to be 1.45 .

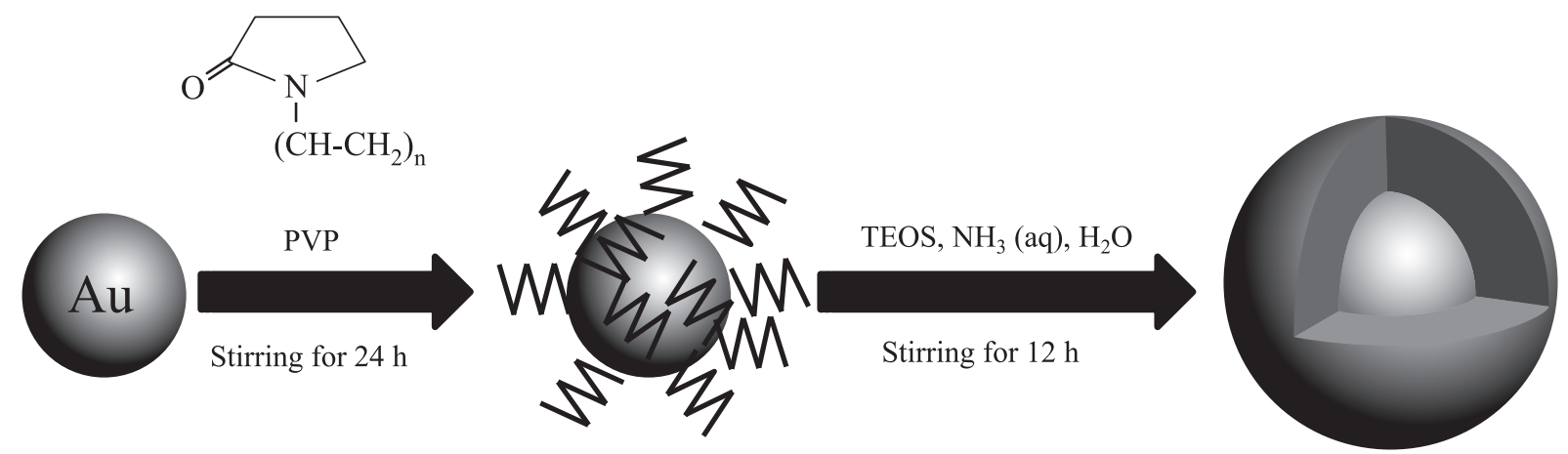

Fig. 1 Schematic of preparation of $\mathrm{Au} @ \mathrm{SiO}_{2}$ nanoparticles. The surface of the Au nanoparticles is modified with PVP followed by the Stöber process for the formation of $\mathrm{SiO}_{2}$ shell. 
Table 1 Composition of the starting solution for fabricating $\mathrm{Au} @ \mathrm{SiO}_{2}$ nanoparticles.

\begin{tabular}{|c|c|c|c|c|c|c|}
\hline $\begin{array}{l}\text { Sample } \\
\text { number }\end{array}$ & $\begin{array}{c}\text { Amount of aqueous } \\
\text { PVP solution, } \\
x(\mathrm{ml})^{\mathrm{a}}\end{array}$ & $\begin{array}{l}\text { Average number of } \\
\text { PVP molecules at } \\
\text { Au surface }\left(\mathrm{nm}^{-2}\right)\end{array}$ & $\begin{array}{c}\text { Amount of } \\
\mathrm{H}_{2} \mathrm{O} \\
y(\mathrm{ml})\end{array}$ & $\begin{array}{c}\text { Amount of } \\
\text { TEOS/ethanol solution, } \\
z(\mathrm{ml})^{\mathrm{b}}\end{array}$ & $\begin{array}{l}\text { Ethanol solution of } \\
\text { Au nanoparticles } \\
(\mathrm{ml})^{\mathrm{c}}\end{array}$ & $\begin{array}{l}\text { Aqueous ammonia } \\
\text { solution }(\mathrm{ml})^{\mathrm{d}}\end{array}$ \\
\hline 1 & 0.50 & 110 & 0.10 & 0.080 & 7.0 & 0.082 \\
\hline 2 & 1.07 & 240 & 0.10 & 0.080 & 7.0 & 0.082 \\
\hline 3 & 1.24 & 280 & 0.10 & 0.080 & 7.0 & 0.082 \\
\hline 4 & 1.50 & 340 & 0.10 & 0.080 & 7.0 & 0.082 \\
\hline 5 & 1.24 & 280 & 0.00 & 0.080 & 7.0 & 0.082 \\
\hline 6 & 1.24 & 280 & 0.05 & 0.080 & 7.0 & 0.082 \\
\hline 7 & 1.24 & 280 & 0.10 & 0.080 & 7.0 & 0.082 \\
\hline 8 & 1.24 & 280 & 0.15 & 0.080 & 7.0 & 0.082 \\
\hline 9 & 1.24 & 280 & 0.10 & 0.040 & 7.0 & 0.082 \\
\hline 10 & 1.24 & 280 & 0.10 & 0.080 & 7.0 & 0.082 \\
\hline 11 & 1.24 & 280 & 0.10 & 0.160 & 7.0 & 0.082 \\
\hline 12 & 1.24 & 280 & 0.10 & 0.240 & 7.0 & 0.082 \\
\hline
\end{tabular}

a The concentration of PVP is $25.6 \mathrm{mg} / \mathrm{ml}$.

$\mathrm{b}$ The concentration of TEOS is $10 \mathrm{vol} \%$.

c The number density of the particles is $8.74 \times 10^{11}$ particles $/ \mathrm{ml}$.

${ }^{d}$ The concentration of ammonia is $28 \mathrm{wt} \%$.

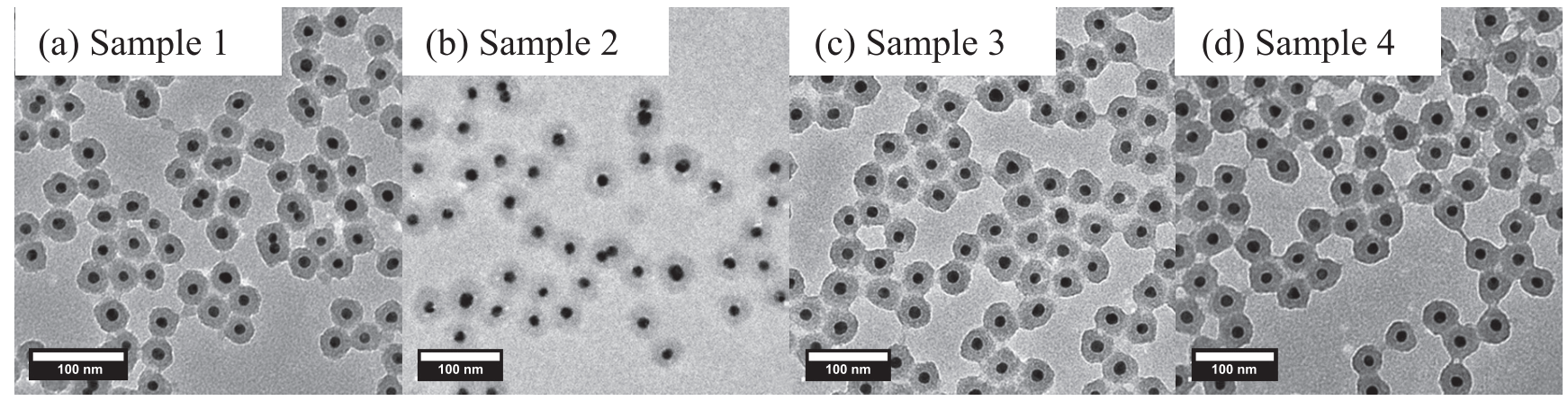

Fig. 2 TEM images of the samples with a varied amount of PVP: (a) $0.5 \mathrm{ml}$, (Sample 1), (b) $1.07 \mathrm{ml}$, (Sample 2), (c) 1.24 ml, (Sample 3), (d) $1.50 \mathrm{ml}$, (Sample 4). Scale bars $=100 \mathrm{~nm}$.

\section{Results and Discussion}

3.1 Influence of the starting composition on the shell morphology

\subsubsection{Influence of PVP}

The role of PVP is to stabilize the Au nanoparticles during the $\mathrm{SiO}_{2}$ shell growth and to provide the Au surface with the affinity to $\mathrm{SiO}_{2}$. We fix the amounts of the other materials and examine the influence of PVP. The amount of aqueous PVP solution $(x)$ is changed as shown in Table 1 and the corresponding TEM images are in Fig. 2. All samples have the similar thickness of the $\mathrm{SiO}_{2}$ shell, but uniformity of the shell varies with the samples. When $x<1.24 \mathrm{ml}$, multiple cores exist in one particle (see Fig. 2a). This is because the surface of Au nanoparticles was not effectively modified with PVP so that attached PVP molecules do not sufficiently shield the large attractive force based on van der Waals force between these particles ${ }^{18)}$. The amount of PVP is enough when $x=1.24 \mathrm{ml}$ which gives rise to a smooth shell compared to the other samples. When $x=$ $1.24 \mathrm{ml}$, there are approximately $280 \mathrm{~nm}^{-2}$ PVP molecules on the Au surface. This is about five times more than that used in the previous paper by Graf et al. ${ }^{18)}$.

3.1.2 Influence of water

We fix the amount of aqueous PVP solution as $1.24 \mathrm{ml}$ 
and examine the effects of the amount of water. The amount of water $(y)$ is changed as shown in Table 1 and the corresponding TEM images are shown in Fig. 3. The results show that the more $y$ is, the thicker the shell is until its amount reaches $0.10 \mathrm{ml}$. This is because water accelerates the hydrolysis and condensation of TEOS. It is noted that the shell grows even though $y=0 \mathrm{ml}$ because the system contains water from aqueous ammonia solution. However, when $y=0.15 \mathrm{ml}$, the shell is thinner than that at $y=0.1 \mathrm{ml}$ because the reaction is so fast that $\mathrm{Au}$-free $\mathrm{SiO}_{2}$ nanoparticles are formed by the homogeneous nucleation of $\mathrm{SiO}_{2}$ in the solution.

\subsubsection{The influence of TEOS}

Lastly, we examine the influence of the amount of TEOS/ethanol solution under the conditions that the amount of aqueous PVP solution is $1.24 \mathrm{ml}$ and the amount of water is $0.1 \mathrm{ml}$. The amount of TEOS/ethanol solution $(z)$ is changed as shown in Table 1 and the corresponding TEM images are shown in Fig. 4. The shell becomes thicker with an increase of the amount of $z$. This is because the amount of shell precursor, i.e., TEOS, is increased. The monotonous increase in shell thickness suggests the absence of homogeneous nucleation of $\mathrm{SiO}_{2}$, which produces $\mathrm{Au}-\mathrm{free} \mathrm{SiO}_{2}$ nanoparticles.
3.1.4 Fine control of shell thickness by the amount of TEOS

The above results suggest that the fine control of the shell thickness is possible by tuning the amounts of water $(y)$ and TEOS/ethanol solution $(z)$ in the starting solution that contains a sufficient amount of PVP. Especially the variation is monotonous with $z$ under the conditions we have adopted as shown in Fig. 5 (a). The shell thickness increases from 8.5 to $21.2 \mathrm{~nm}$ when $z$ increases from 0.04 to $0.24 \mathrm{ml}$. Fig. 5 (b) shows that the shell volume per particle increases in roughly proportion to the amount of TEOS. The shell volume is about $1 / 8$ of the value when all the TEOS is converted to $\mathrm{SiO}_{2}$. This discrepancy indicates that the reactions are not completed in $12 \mathrm{~h}$ so that it would be possible to tune the shell thickness by the reaction time.

3.2 Extinction properties of $\mathrm{Au}$ and $\mathrm{Au} @ \mathrm{SiO}_{2}$ nanoparticles

In order to examine the optical quality of the core-shell nanoparticles prepared, we picked up several samples and measured optical extinction spectra. Fig. 6 (a) is the extinction spectra of $\mathrm{Au}$ nanoparticles and $\mathrm{Au} @ \mathrm{SiO}_{2}$ nanoparticles dispersed in water. As the shell thickness is varied from 0 to $25 \mathrm{~nm}$, the extinction peak is slightly redshifted from 519 to $524 \mathrm{~nm}$. This is because the refractive index around Au nanoparticles is changed due to the

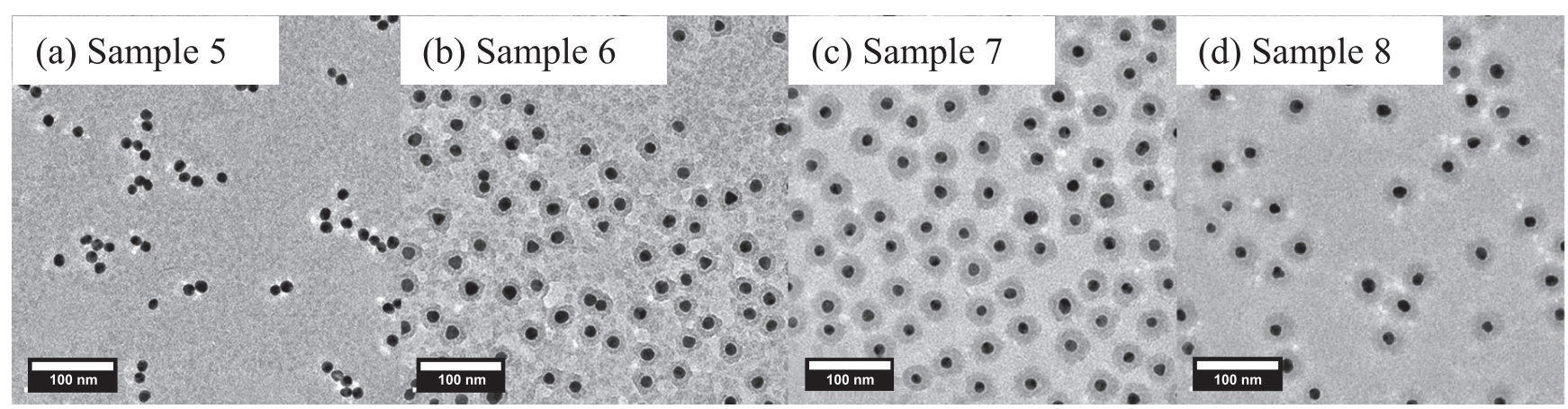

Fig. 3 TEM images of the samples with varied amount of water: (a) $0.00 \mathrm{ml}$, (Sample 5), (b) $0.05 \mathrm{ml}$, (Sample 6), (c) 0.10 ml, (Sample 7), (d) $0.15 \mathrm{ml}$, (Sample 8). Scale bars $=100 \mathrm{~nm}$.

(a) Sample 9

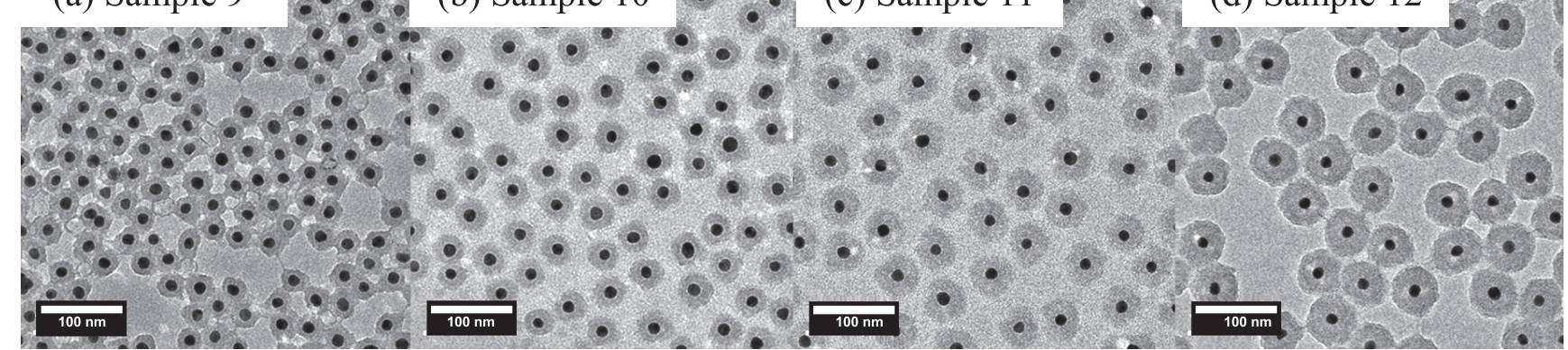

Fig. 4 TEM images of the samples with varied amount of 10 vol\% TEOS/ethanol solution: (a) $0.040 \mathrm{ml}$, (Sample 9), (b) 0.080 ml, (Sample 10), (c) $0.160 \mathrm{ml}$, (Sample 11), (d) $0.240 \mathrm{ml}$, (Sample 12). Scale bars $=100 \mathrm{~nm}$. 

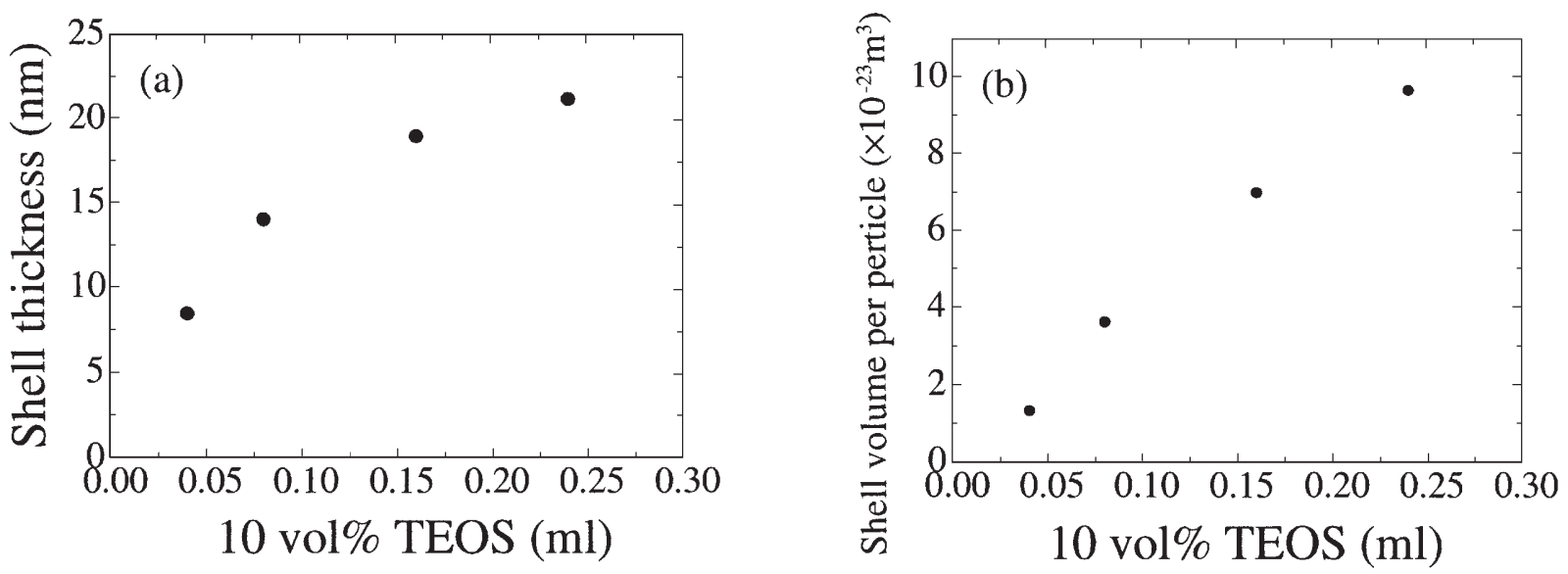

Fig. 5 Variation of the shell thickness (a) and of the shell volume per particle (b) with the amount of TEOS. Note that the concentration of TEOS is $10 \mathrm{vol} \%$ in ethanol.
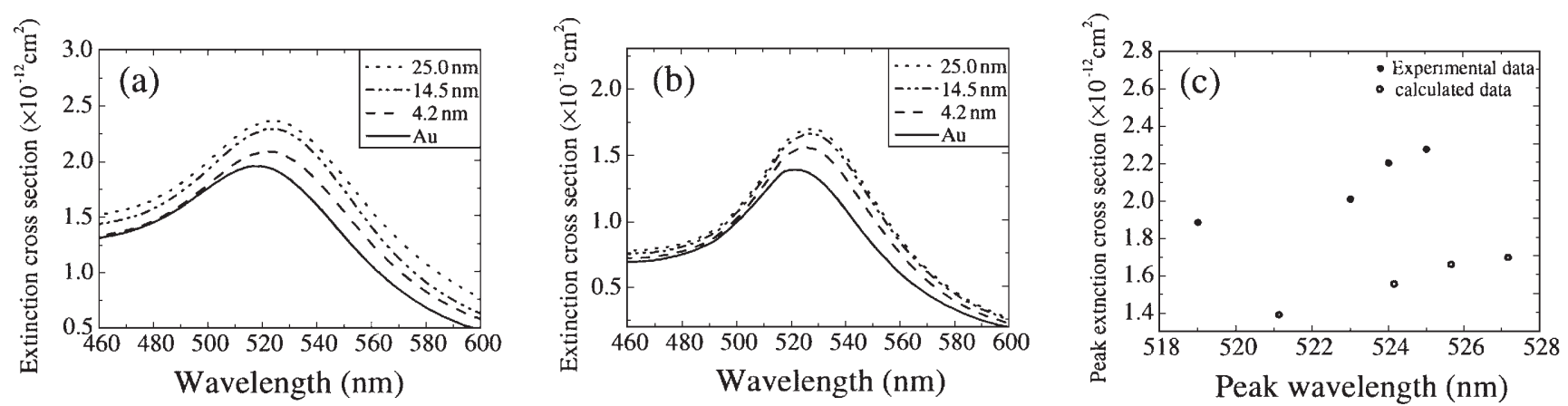

Fig. 6 (a) Measured extinction spectra of Au nanoparticles and $\mathrm{Au} @ \mathrm{SiO}_{2}$ nanoparticles. (b) The extinction cross section of $\mathrm{Au}$ and $\mathrm{Au} @ \mathrm{SiO}{ }_{2}$ nanoparticles calculated by DDSCAT. (c) The variation of the peak wavelength and corresponding extinction cross section extracted from the experimental and calculated spectra.

presence of $\mathrm{SiO}_{2}$ coating. It should be noticed that the thicker the shell is, the larger the extinction cross section is. This is mainly because the geometrical cross section is increased with the shell thickness. The results are compared to the simulation results based on DDA. The simulated spectra are shown in Fig. 6(b), and Fig. 6 (c) shows the peak wavelength and corresponding cross section extracted from the simulated and experimental spectra. Although the values of cross section derived from the simulation are not exactly the same as those obtained experimentally, we can reproduce the red-shift of the peak with an increase of shell thickness without using any adjustable fitting parameters. The experimental spectra are broader compared to the simulated spectra, because of the size distribution of $\mathrm{Au}$ nanoparticles prepared experimentally.

\section{Conclusions}

We have developed a method to tune the shell thickness of Au@ $\mathrm{SiO}_{2}$ nanoparticles. The typical shell thickness is below $25 \mathrm{~nm}$, which is useful for plasmonic applications.
With the optimal amount of PVP absorbing onto the surface, the Au nanoparticles become stable in both water and ethanol. By changing the amounts of water and TEOS in the solution, we can control the surface smoothness and thickness of the $\mathrm{SiO}_{2}$ shell. The extinction cross section of the $\mathrm{Au} @ \mathrm{SiO}_{2}$ nanoparticles is well reproduced by the simulation.

\section{Acknowledgments}

This study was supported by a Grant-in-Aid for Scientific Research (B, No. 24350104 and C, No. 24560824) and for Challenging Exploratory Research (No. 24656385) from the Ministry of Education, Culture, Sports, Science and Technology (MEXT), Japan.

\section{References}

1) M.A. Noginov, G. Zhu, A.M. Belgrave, R. Bakker, V.M. Shalaev, E.E. Narimanov, S. Stout, E. Herz, T. Suteewong, U. Wiesner: "Demonstration of a spaserbased nanolaser", Nature, 460(2009) 1110-1113. 
2) M.D. Brown, T. Suteewong, R.S.S. Kumar, V. D’Innocenzo, A. Petrozza, M.M. Lee, U. Wiesner, H.J. Snaith: "Plasmonic Dye-Sensitized Solar Cells Using Core-Shell Metal-Insulator Nanoparticles", Nano Lett., $11(2011) 438-445$.

3) J.F. Li, Y.F. Huang, Y. Ding, Z.L. Yang, S.B. Li, X.S. Zhou, F.R. Fan, W. Zhang, Z.Y. Zhou, D.Y. Wu, B. Ren, Z.L. Wang, Z.Q. Tian: "Shell-isolated nanoparticle-enhanced Raman spectroscopy", Nature, 464(2010)392-395.

4) C.J. Orendorff, A. Gole, T.K. Sau, C.J. Murphy: "Surface-Enhanced Raman Spectroscopy of SelfAssembled Monolayers: Sandwich Architecture and Nanoparticle Shape Dependence", Anal. Chem., 77 (2005)3261-3266.

5) C.J. Murphy, T.K. Sau, A.M. Gole, C.J. Orendorff, J. Gao, L. Gou, S.E. Hunyadi, T. Li: "Anisotropic Metal Nanoparticles: Synthesis, Assembly, and Optical Applications", J. Phys. Chem. B, 109(2005) 1385713870

6) L. Wang, C. Clavero, Z. Huba, K.J. Carroll, E.E. Carpenter, D. Gu, R.A. Lukaszew: "Plasmonics and Enhanced Magneto-Optics in Core-Shell $\mathrm{Co}-\mathrm{Ag}$ Nanoparticles", Nano Lett., 11(2011)1237-1240.

7) P. Bharadwaj, L. Novotny: "Spectral dependence of single molecule fluorescence enhancement", Opt. Express, 15(2007) 14266-14274.

8) O.G. Tovmachenko, C. Graf, D.J. van den Heuvel, A. van Blaaderen, H.C. Gerritsen: "Fluorescence Enhancement by Metal-Core/Silica-Shell Nanoparticles", Adv. Mater., 18(2006)91-95.

9) X. Meng, K. Fujita, S. Murai, T. Matoba, K. Tanaka: "Plasmonically Controlled Lasing Resonance with Metallic-Dielectric Core-Shell Nanoparticles", Nano Lett., 11(2011)1374-1378.

10) A.P. Philipse, M.P.B. van Bruggen, C. Pathmamanoharan: "Magnetic silica dispersions: preparation and stability of surface-modified silica particles with a magnetic core", Langmuir, 10(1994)92-99.

11) I. Pastoriza-Santos, J. Pérez-Juste, L.M. Liz-Marzán: "Silica-Coating and Hydrophobation of CTABStabilized Gold Nanorods", Chem. Mater., 18(2006) 2465-2467.

12) D.K. Yi, S.T. Selvan, S.S. Lee, G.C. Papaefthymiou, D. Kundaliya, J.Y. Ying: "Silica-Coated Nanocomposites of Magnetic Nanoparticles and Quantum Dots", J. Am. Chem. Soc., 127 (2005)4990-4991.

13) D.K. Yi, S.S. Lee, J.Y. Ying: "Synthesis and Applications of Magnetic Nanocomposite Catalysts", Chem. Mater., 18(2006) 2459-2461.

14) S.T. Selvan, T.T. Tan, J.Y. Ying: "Robust, NonCytotoxic, Silica-Coated CdSe Quantum Dots with Efficient Photoluminescence", Adv. Mater., 17(2005) 1620-1625.

15) L.M. Liz-Marzán, P. Mulvaney: "The Assembly of Coated Nanocrystals", J. Phys. Chem. B, 107(2003) 7312-7326.

16) W. Stöber, A. Fink: "Controlled Growth of Monodisperse Silica Spheres in the Micron Size Range", J. Colloid Interface Sci., 26(1968)62-69.

17) L.M. Liz-Marzán, M. Giersig, P. Mulvaney: "Synthesis of Nanosized Gold-Silica Core-Shell Particles", Langmuir, 12(1996)4329-4335.

18) C. Graf, D.L.J. Vossen, A. Imhof, A. van Blaaderen: "A General Method To Coat Colloidal Particles with Silica", Langmuir, 19(2003)6693-6700.

19) E. Mine, A. Yamada, Y. Kobayashi, M. Konno, L.M. Liz-Marzán: "Direct coating of gold nanoparticles with silica by a seeded polymerization technique", J. Colloid Interface Sci., 264(2003)385-390.

20) Y. Han, J. Jiang, S.S. Lee, J.Y. Ying: "Reverse Microemulsion-Mediated Synthesis of Silica-Coated Gold and Silver Nanoparticles", Langmuir, 24(2008) 5842-5848.

21) P. Botella, A. Corma, M.T. Navarro: "Single Gold Nanoparticles Encapsulated in Monodispersed Regular Spheres of Mesostructured Silica Produced by Pseudomorphic Transformation", Chem. Mater., 19 (2007) 1979-1983.

22) G. Frens: "Controlled nucleation for the regulation of the particle size in monodisperse gold solutions", Nat. Phys. Sci., 241(1973)20-22.

23) B.T. Draine, P.J. Flatau: "Discrete-dipole approximation for scattering calculations", J. Opt. Soc. Am. A, 11 (1994) 1491-1499.

24) B.T. Draine, P.J. Flatau: "The discrete dipole approximation for periodic targets: I. theory and tests", JOSA A., 25(2008)2693-2703.

25) P.B. Johnson, R.W. Christy: "Optical Constants of the Noble Metals", Phys. Rev. B, 6(1972)4370-4379. 